Wright State University

CORE Scholar

Kno.e.sis Publications

The Ohio Center of Excellence in Knowledge-

Enabled Computing (Kno.e.sis)

9-1997

\title{
From Contemporary Workflow Process Automation to Adaptive and Dynamic Work Activity Coordination and Collaboration
}

Amit P. Sheth

Wright State University - Main Campus, amit@sc.edu

Follow this and additional works at: https://corescholar.libraries.wright.edu/knoesis

Part of the Bioinformatics Commons, Communication Technology and New Media Commons, Databases and Information Systems Commons, OS and Networks Commons, and the Science and Technology Studies Commons

\section{Repository Citation}

Sheth, A. P. (1997). From Contemporary Workflow Process Automation to Adaptive and Dynamic Work Activity Coordination and Collaboration. Proceedings of the Eighth International Workshop on Database and Expert Systems Applications, 24-27.

https://corescholar.libraries.wright.edu/knoesis/860

This Conference Proceeding is brought to you for free and open access by the The Ohio Center of Excellence in Knowledge-Enabled Computing (Kno.e.sis) at CORE Scholar. It has been accepted for inclusion in Kno.e.sis Publications by an authorized administrator of CORE Scholar. For more information, please contact librarycorescholar@wright.edu. 


\title{
From Contemporary Workflow Process Automation to Adaptive and Dynamic Work Activity Coordination and Collaboration
}

\author{
Amit Sheth \\ Large Scale Distributed Information Systems Lab \\ University of Georgia \\ 415 GSRC, Athens, GA 30602-7404 USA \\ email: amit@cs.uga.edu,URL:http://lsdis.cs.uga.edu
}

\begin{abstract}
This position paper outlines a research agenda for researchers in the area of workflow. We believe that today's workflow systems should evolve to what is termed as work coordination and collaboration systems (WCCSs). A WCCS will (a) adapt to various changes in the organization (including its interactions with external organizations) and the organizational processes by being able to change the processes definitions as well as change the processes and component activities while they are being enacted or executed, and (b) support a unified framework for managing coordination, collaboration, and information-based decision making activities that naturally occur as part of organizational processes. A WCCS can then not only support automation of the routine and well defined processes, but also support better human involvement and manage more complex, dynamic, and higher value mission critical processes. This can lead to significantly improved productivity and quality of results. It is further suggested that a multidisciplinary approach is an essential element of the part toward developing WCCSs.
\end{abstract}

\section{A Look Beyond the Contemporary Workflow Technology}

The central issue we wish to address in this paper is, what are the key research and technological issues as we look beyond current research and practices in managing workflow processes (as perceived by the current technology and products) to a more comprehensive notion of support for organizational processes. Let's start with the relevant definitions (from the American Heritage Dictionary). An organization is defined as a number of persons or groups having specific responsibilities and are unified for a particular purpose. A process is defined as a series of actions, changes, or functions that bring about an end or result. As we will argue, the workflow processes, as typically perceived today and supported by contemporary technology, address only part of the issues involved in supporting processes that occur in organizations, or organizational processes. The essential elements missing include more comprehensive participation of humans and the dynamic nature of many organizations and organizational processes.

Next only to the Internet related technology and products, the workflow technology and products are arguably the most influential new breed of software systems from the perspective of achieving significant impact on commercial organizations. With over $\$ 2$ billion in annual revenue related to workflow products and services in 1996, this marketplace has grown at a significant pace. Many organizations have shifted their data-centric apporach in the context of the information systems technology and solutions to a processcentric one (albeit some times going to extremes, rather than considering these as two sides of a coin). However, the focus of the state-of-the-art workflow technology has been arguably limited to routine processes and coordinating execution of relatively simple activities. The emphasis has been largely on office automation and other mostly routine processes as may be supported with activities involving email, forms based data access, documents and imaging, and some times a broader range of activities supported by (legacy) information systems. Figure 1 shows a variety of technological and market segment based approaches to develop workflow products.

We would argue that the current technology can support a majority (may be $70 \%$ to $80 \%$ ) of all coordination-centric processes in many typical organizations. So what is missing, and what relevant research is called for?

First, some of the more complex, mission-critical, and consequently more valuable organizational processes cannot be supported well with the current tech- 


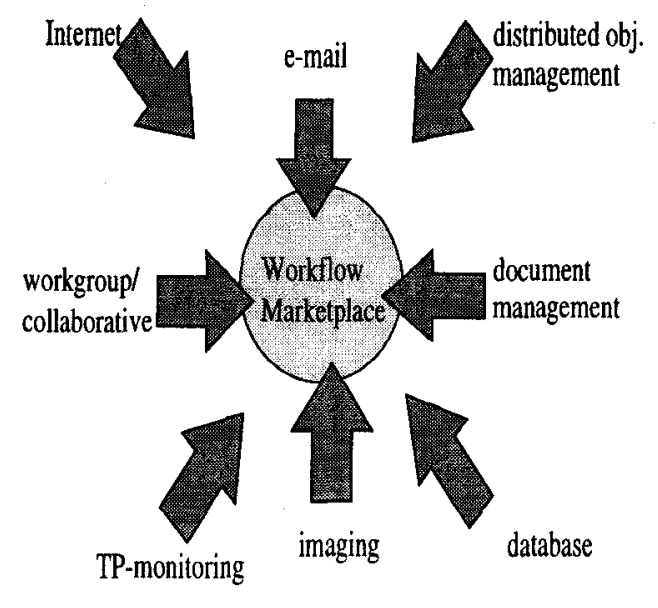

Figure 1: Workflow marketplace relevant technologies and markets

nology and products. Second, coordination by itself is not adequate to support many organizational processes. Our own practical experience at the LSDIS lab and its industry partners with the applications of our coordination-oriented METEOR (Managing EndTo-End OpeRations) workflow management system (WFMS) and collaboration-oriented CaTCH (Collaborative TeleConsulting for Healthcare) system show that many organizational processes are neither limited to coordination nor collaborative activities alone. For example, when using a METEOR WFMS supported application for supporting a coordination dominated clinical process, a physican might wish to consult (hence collaborate with) a specialist when $\mathrm{s} / \mathrm{he}$ comes across abnormal lab results not within the range of her/his normal expertise.

With a somewhat expanded view of organizational processes elluded to above, many research communities are addressing various aspects of organizational processes. These include, software process, software engineering, database, management information systems, CSCW/groupware, distributed computing, cooperative information systems, human computer interactions, distributed AI, software (esp. collaborative) agents, organizational sciences, and others. For a technological perspective, we have seen two relevant and significant advances in the past few years, one in the area of distributed computing, and the other in information integration. Our ability to achieve software integration in distributed environments has substantially improved. Web and distributed object management (especially CORBA), among many other infrastructural and standards-based technologies are largely responsible for this. These integration capa- bilities have also been utilized to develop the corresponding ability to integrate data or information from multiple sources. Many component technologies relevant to support organizational processes have either matured (databases, groupware, etc.) or have become viable for operational (albeit sometime limited) use (e.g., desktop videoconferencing and application sharing, whiteboarding and other collaboration software, etc.).

Some of the features that are exhibited by individual component technologies, such as support for scalability, transactional properties, error handling and automatic recovery in database management systems, need to be carried over to the process arena. Many similar items in the wish-list can be included here that benefit from different research areas and component technologies (see [SGJ96] for several of these). However, more fundamental issues that now deserve attention include the ways to:

- more effectively involve humans and human intensive (e.g., decision making) activities in the computer supported organizational processes (or alternatively work processes, where the delibrate exclusion of "flow" implies that the processes are more fluid, more dynamic)

- support processes that are more flexible, pliable, or adaptive, and hence dynamically alterable, such that the computer supported organizational processes can support more of the intelligent and intuitive support that humans orchestrating and participating in an organizational process can provide

The main goal here is not only to take away from humans the more routine activities and automate or support workflow processes that require coordination, but to support them in processes and activities that involve coordination, collaboration, as well as decision making. In other words, we propose that a more comprehensive approach to supporting organizational processes should include integrated support for coordination, collaboration, and management of information (see Figure 2). Included in the range of coordination and collaboration mode are asynchronous as well as synchronous collaborations. The information management component also includes integrated access to distributed and heterogeneous data and media, as well as the information processing software (for analysis, mining, filtering, aggregation or fusion of information) that support in decision making.

The corollary to the above is that currently disperate technologies for workflow automation or process 


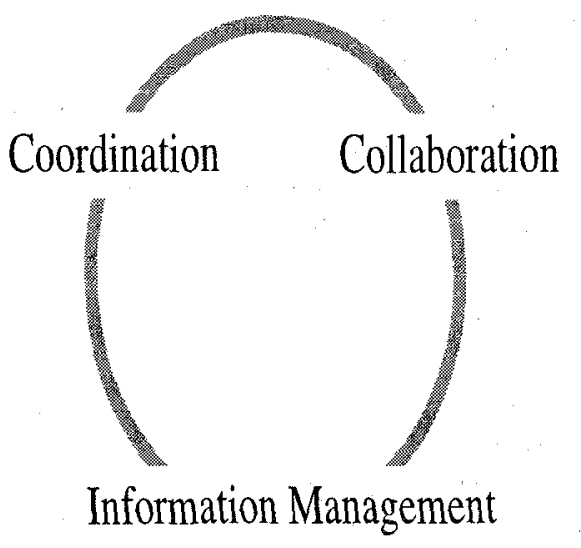

Figure 2: Components of a more unified solution

management (for coordination), group/team support and CSCW, (desktop) conferencing and collaboration, and information management, will need to be integrated. Hopefully this will happen within the context of a broader and more unified modeling and enactment/orchestration framework with adequate attention to all key components (for coordination, collaboration, and information management), rather than as a patchwork, interfacing, or interoperability of independent technologies.

\section{Dynamic and Adaptive Work Activ- ity Coordination and Collaboration}

Dynamic behavior that identifies, understands and adapts to the changes is fundamental to the coordination and collobarations involving human and computer performed work activities in support of the organizational processes. We envision adaptive and dynamic Work Coordination and Collaboration Systems (WCCSs) that support the corresponding requirements of organizational processes. Key requirements and influencing factors of a WCCS include the following.

- Many of the organizational processes are not static, and some aspects of their specifications can only be supplied during their enactments (or runtime).

- Processes change continually for an organization to remain competitive in the market, to meet customer's needs or the external requirements such as regulations, to support current trends in improving the speed and quality of services, to react to external or internal events, to benefit from changes in technology, and other reasons leading to process reengineering.
- The organizations themselves change for many reasons, such as changing in personnel, refocusing of business strategy, new partnerships and mergers or acquisitions, and more interesting, by forming virtual corporations. A very important issue here includes the changing relationships and interfaces with other organizations (including partners, contractors, supplies, customers, etc.)

A WCCS may adapt to the changes of the types indicated above in several ways. These include changes in the process model or specification (including individual activities, relationships or dependencies between activities or other constraints, and reusing existing or adding new process components) and changes in execution environments including the changes in entities participating in the processes. A partial listing of a WCCS capabilities include [SK97]:

- support for dynamic processes that can change at run time automatically or with human input. Here, dynamic refers to: a) the ability of the run-time model of the execution engine (or enactment system) to change course automatically to support a newly-specified next activity in the work process execution, b) work processes with automated application activities (tasks) as well as activities involving human involvement with structured or unstructured collaboration among participants, c) concurrent execution of (sub)processes corresponding to interactions among different activities;

- a high degree process reuse, using a repository for consistency of process ontology, resource ontology (including user-centered ontologies such as organizational roles, authorizations, user profiling, and other context required to create the appropriate views of information in a particular task), work process definitions, and detailed activity specifications;

- collaboration, providing a variety of tools (voice, video, whiteboarding) supporting human interaction, both at the individual activity level and the work process management level;

- adaptive work processes, using monitoring agents (also called sentinels), or other appropriate agents, that react to changes in the relevant information resources or the environment, fuse the appropriate information, and notify the work coordination and collaboration processes. Here, adaptablility includes to the ability of the WCCS 
to monitor, interpret, and react to rapidly changing information sources;

Additional thoughts on the WCCS, including additional WCCS capabilities such as visualization and security, appear in [SK97].

\section{A Multidisciplinary Agenda}

We believe much of what needs to be done would require a multidisciplinary approach. This echos the quote given below from the final report written by a multidisciplinary team of researchers following the recent NSF workshop on Workflow and Process Automation in Information Systems [SGJ96]:

\begin{abstract}
"Work Activity Coordination" involves such multidisciplinary research and goes beyond the current thinking in contemporary workflow management and Business Process Reengineering (BPR). In particular, instead of perceiving problems in prototypical terms such as the information factory, white-collar work and bureaucracy, we believe that this limited point of view can be explained by a lack of synergy between organizational science, methodologies, and computer science. Multidisciplinary research projects, based on mutual respect and willingness to learn from another discipline, can help to create a thriving research community that builds upon the strengths of different disciplines, such as distributed systems, database management, software process management, software engineering, organizational sciences, and others."
\end{abstract}

I welcome the researchers and practioners interested in participating in this multidisciplinary agenda to meet us at the NSF-sponsored virtual gathering place at http://lsdis.cs.uga.edu/worp.

\section{Acknowledgements}

Discussions held at the NSF Workshop on Workflow and Process Automation in Information (http://lsdis.cs.uga.edu/NSF-workflow) and regular discussions with the METEOR team, especially my colleagues Krys Kochut and John Miller, and my students Zongwei Luo and Devashish Worah, have influenced the views expressed in this paper. This work is partially supported by the NIST Advanced Technology Program in Healthcare Information Infrastructure Technology (under the HIIT contract, number 70NANB5H1011) in partnership with Healthcare Open Systems and Trials (HOST) consortium.

\footnotetext{
${ }^{1}$ and collaboration
}

\section{References}

[SGJ96] A. Sheth, D. Georgakopoulos, S. Joosten, M. Rusinkiewicz, W. Scacchi, J. Wileden, and A. Wolf. Report from the NSF Workshop on Workflow and Process Automation in Information Systems. Technical report, University of Georgia, UGA-CS-TR-96-003, July 1996 (also in ACM SIGMOD Record, September 1996).

URL:

LSDIS.cs.uga.edu/activities/NSFworkflow.

[SK97] A. Sheth and K. Kochut. Workflow Applications to Research Agenda: Scalable and Dynamic Work Coordination and Collaboration Systems. In Proceedings of the NATO Advanced Study Institute on workflow Management Systems and Interoperability, Istambul, Turkey, August 12-21, 1997. 